\title{
An osteoblast origin for craniofacial dysplasia in neurofibromatosis type I
}

\begin{abstract}
Sphenoid wing dysplasia (SWD), which results in a craniofacial deformity, is the third most common skeletal deformity in Neurofibromatosis Type I (NF1) patients. A neurofibromin osteoblast conditional deletion mouse model (Nflob-/-) has been developed to study the skeletal abnormalities in NF1. No overt morphological phenotype is seen in the appendicular or axial skeleton of these mice. Nflob-/- mice have been shown to have increased bone porosity and lower bone density. We noted a progressive craniofacial deformity, which results in cranial asymmetry, malocclusion and unilateral proptosis of an eye. To assess this deformity, we employed micro-computed tomography $(\mathrm{mCT})$ and geometric morphometric analysis to compare Nflob-/-mouse skulls to control animals. Landmarks were placed on the images at 13 biologically relevant cranial anatomical sites. Analysis of distances and angles between these landmarks revealed that there is significant variation between the Nflob-/- mice and controls. We found that the nasal and frontal bones of Nflob-/- mice skulls are deviated from the central line, whereas it was straight in controls. The nasal region is tipped downward in Nflob-/- mice. We also noted that the cranium shows a trend toward macrocephaly in Nflob-/- mice whereas other measurements were within the range of normal control mice. These differences correspond to those seen in craniofacial dysplasia and SWD in NF1 patients. No tumors were found associated with the craniofacial dysplasia in Nflob-/- mice. Our results identify a primarily osteoblast origin for the pathogenesis of craniofacial dysplasia in Nflob-/- mice and strongly support an osteoblast origin for SWD in NF1. In addition, we identify and validate a novel mouse model with which to explore molecular mechanisms and test preventative treatments for SWD in NF1.
\end{abstract}

Keywords: sphenoid bone, craniofacial abnormalities, malocclusion, megalencephaly, facial asymmetry, exophthalmos
Volume 6 Issue 6 - 2017

\author{
Ibraheem K Bamaga,' Regina PO Sullivan, ${ }^{2}$ \\ James Schmitz, ${ }^{3}$ Weston Fath, ${ }^{3}$ Roberto J \\ Fajardo, ${ }^{3}$ Kevin P McHugh ${ }^{4}$ \\ 'School of Dentistry, University of Florida, USA \\ ${ }^{2} \mathrm{PBC}$ BioMed, Europe \\ ${ }^{3}$ Department of Orthopaedics, University of Texas Health \\ Science Center at San Antonio, USA \\ ${ }^{4}$ Department of Periodontics, University of Florida College of \\ Dentistry, USA
} University of Florida, 1395 Center Dr. Room DI0-28, Gainesville, Florida, 32610, USA, Tel 3522153120 Email Dr_ibamaga@yahoo.com

Received: March 23, 2017 | Published: April 06, 2017
Abbreviations: NF1, neurofibromatosis type 1; SWD, sphenoid wing dysplasia; mCT, micro computed tomography; EDMA, euclidean distance matrix analysis; PNF, plexiform neurofibromas

\section{Introduction}

Neurofibromatosis type 1 (NF1) is one of the most frequent human genetic disorders, with an incidence at birth of approximately one in 3000 to one in $3500 .{ }^{1} \mathrm{NF} 1$ is characterized by the predisposition to benign and malignant tumors of the peripheral and central nervous system. NF1 patients may also exhibit noncancerous symptoms such as the presence of café-au-lait macules, axial freckling, learning disabilities, and skeletal dysplasia. ${ }^{2,3}$ Clinically, NF1 patients exhibit skeletal congenital or developmental abnormalities, resulting in alterations in bone shape or size. Skeletal defects can occur in the cranium, ribs, long bones, or the spine. ${ }^{4}$ These manifestations often worsen with age and in some cases have been associated with loss of heterozygosity at the NF1 locus. ${ }^{5,6}$ In a significant number of NF1 patients, orthopedic intervention results in failure of correction due to poor bone healing and may reflect a generalized defect in bone metabolism. ${ }^{4}$ The skeletal involvement affects approximately half of all NF1 patients. ${ }^{7-9}$ Craniofacial involvement, which includes macrocephaly, sphenoid wing dysplasia (SWD), short mandible and maxilla, cranial base deformity and malocclusion is seen in $11-20 \%$ of patients with NF1. ${ }^{10,11}$ Other craniofacial findings have been reported in NF1 including a widening of inferior alveolar canal, an enlarged mandibular foramen, and a reduction in mandibular angle. In rare cases plexiform Neurofibromas have been reported to be associated with impacted teeth and deformed alveolar ridge while optic nerve gliomas have been associated with orbit defects. ${ }^{12-15}$ NF1 patients may also display generalized defects in bone metabolism and bone turnover, resulting in osteoporosis in up to one half of affected individuals. ${ }^{16,17}$ Recently a neurofibromin osteoblast conditional knockout mouse was developed to study the skeletal manifestations of NF1. In this model (Nflob-/-) the loxP-flanked neurofibromin gene is conditionally deleted in bone-forming osteoblasts and osteoblast lineage cells under the control of $2.3 \mathrm{~kb}$ collal promotor-driven Cre recombinase. ${ }^{18,19}$ The Nflob-/- mouse was previously shown to develop a bone mass phenotype including high bone porosity with increased osteoclast and osteoblast numbers, as seen in NF1 patients. However, Nflob-/- mice display none of the appendicular or axial skeletal dysplasia seen in $\mathrm{NF} 1 .^{20}$ We observed a high rate of malocclusion in Nf1ob-/- animals as they age compared with animals of the same genetic background. Upon closer inspection, unilateral proptosis of an eye was apparent as was facial asymmetry. In this study we use morphometric analysis to characterize the craniofacial dysplasia in Nflob-/- mice and compare it to SWD and craniofacial dysplasia in NF1. Additionally, we establish an osteoblast lineage origin for the craniofacial dysplasia in the Nflob-/-mouse and identify a mouse model with which to test treatments aimed at slowing or blocking progression of sphenoid wing bone and craniofacial dysplasia in NF1 patients. 


\section{Materials and methods}

\section{Animal husbandry and mouse models}

Nflflox/flox (Nf1fl/fl) mice were kindly provided by Florent Elefteriou et al. ${ }^{19}$ Transgenic B6.FVB-Tg (Col1a1-cre) 1 Kry mice, ${ }^{18}$ were purchased from Riken (RBCR_NO. RBRC05603). Nflob-/mice were generated by crossing Nf1fl/fl with Nflfl/fl; Col1a1cre mice. The genetic backgrounds of mice were assessed by the DartMouse ${ }^{\mathrm{TM}}$ Speed Congenic Core Facility at the Geisel School of Medicine at Dartmouth. Dart Mouse employed the Golden Gate Genotyping Assay (1llumina Inc, San Diego, CA) to interrogate 1449 SNPs spread throughout the genome. The raw SNP data were analyzed using Dart Mouse's SNaP-Map ${ }^{\mathrm{TM}}$ and Map-Synth ${ }^{\mathrm{TM}}$ software, allowing the determination of the genetic background at each SNP location. The background test revealed that all mouse strains used in this study were fully back-crossed onto the C57BL/6 background. Genotypes of individual mice were determined by polymerase chain reaction (PCR) using DNA from tail tip at weaning. All mice were maintained in a specific-pathogen-free barrier facility with free access to food (Envigo Teklad Global diet 2918) and water. All procedures were approved by the Institutional Animal Care and Use Committee.

\section{Genotyping}

For genotyping, DNA was extracted from $0.5 \mathrm{~cm}$ tail snip using the DNeasy blood and tissue kit (Qiagen, cat\# 69506) with final elution volume of $200 \mathrm{ml}$. Genotyping the $\mathrm{Nf1} / \mathrm{fl} / \mathrm{fl}$ mice was carried out by PCR using three primers to detect WT and Flox alleles. Two primers from intron 30 of the Nf1 gene (P1 and P3) detect a 480-bp band from the WT allele, a third primer (P4) from neo gene with (P1) amplify a 350bp band from the flox allele. PCR was performed under the following conditions: PCR master mix: $3.0 \mathrm{mM} \mathrm{MgCl}_{2}, 0.4 \mathrm{mM}$ dNTPs (Apex Bioresearch Products Cat\# 42-138), $0.2 \mathrm{mM}$ of $\mathrm{P} 1$ primer and $0.4 \mathrm{mM}$ of primers $\mathrm{P} 3$ and $\mathrm{P} 4,0.5 \mathrm{ml}$ of DNA; and the program: 1 cycle of $94^{\circ} \mathrm{C}$ for $5 \mathrm{~min} ; 32$ cycles of $94^{\circ} \mathrm{C}$ for $1 \mathrm{~min} ; 56^{\circ} \mathrm{C}$ for $1 \mathrm{~min}$; and $72^{\circ} \mathrm{C}$ for $1 \mathrm{~min}$; and followed by $72^{\circ} \mathrm{C}$ for $10 \mathrm{~min}$. Genotyping for the Cre transgene was carried out under the following conditions: PCR master mix: $3.0 \mathrm{mM} \mathrm{MgCl}, 0.4 \mathrm{mM}$ dNTPs and 0.05 units $/ \mathrm{ml}$ Apex Taq RED DNA polymerase (Apex Bioresearch Products Cat\# 42-138), $0.4 \mathrm{mM}$ of primers $\mathrm{Cre} 1$ and $\mathrm{Cre} 2$; and the program: 1 cycle of $94^{\circ} \mathrm{C}$ for $5 \mathrm{~min}$; 30 cycles of $94^{\circ} \mathrm{C}$ for $30 \mathrm{sec} ; 56^{\circ} \mathrm{C}$ for $30 \mathrm{sec}$; and $72^{\circ} \mathrm{C}$ for $30 \mathrm{sec}$; and followed by $72^{\circ} \mathrm{C}$ for $10 \mathrm{~min}$. PCR assay for detecting Cre-mediated recombination and completion of deletion was as following: Two primers ( $\mathrm{P} 1$ and $\mathrm{P} 2)$ were used to detect a 280-bp band that resulted from Cre-mediated recombination in the $\mathrm{Nflfl} / \mathrm{fl}$ allele. The four primers $\left(5^{\prime}\right.$ to $\left.3^{\prime}\right)$ are: P1, CTTCAGACTGATTGTTGTACCTGA; $\mathrm{P} 2$, CATCTGCTGCTCTTAGAGGAACA; P3, ACCTCTCTAGCCTCAGGAATGA; P4, TGATTCCCACTTTGTGGTTCTAAG; Cre1, CATGTTTAGCTGGCCCAAATGTTGCTG; and Cre2, CGACCATGCCCAA GAAGAAGAGGAAGGTG. All primers were synthesized by Eurofins Genomics (Ebersberg, Germany).

\section{Preparation of skeleton and landmark data collection}

Mice were sacrificed and skulls were skinned and saved in $70 \%$ ethanol until ready for scanning. Micro computed tomography $(\mathrm{mCT})$ scans were performed for all mice skulls using SKYSCAN 1173 exvivo micro-CT from Bruker Company (Bruker, Kontich, Belgium). Samples were scanned at high resolution and the scans were reconstructed using NRecon software interface (v.1.6.4.6, Bruker micro-CT, Kontich, Belgium). The scan settings were the following: Source Voltage: $60 \mathrm{kV}$, Source Current: 167mA, Image Pixel Size: 13.60mm, Camera: 2K, Filter: Al 0.5mm, Exposure: $2100 \mathrm{~ms}$, Rotation step: 0.7 degrees, Frame Average: 5. The reconstructions were performed using a Feldkamp algorithm and a 5th order polynomial to correct for beam hardening in the images. All scanning and reconstruction parameters were kept constant for all skulls. All resliced and reconstructed scans were transferred to Analyze 10.0ã software (Analyze Direct, Overland Park, KS, USA) for 3D rendering. Three-dimensional coordinate locations of 13 biologically relevant cranial landmarks were recorded (Figure 1). ${ }^{21}$ Detailed description of these landmarks is provided on the landmarks collection page at the Richtsmeier laboratory website, (Table 1). Landmarks are located according to the three orthogonal planes of the $3 \mathrm{D}$ reconstruction of the specimen.
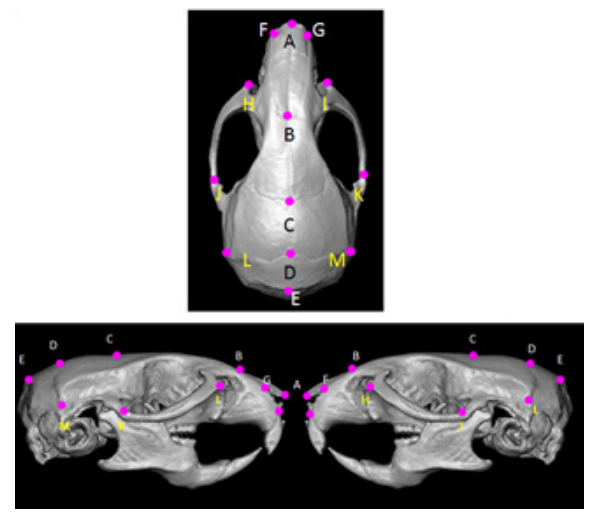

Figure I Mouse skull and landmarks. (a: superior; b: lateral) Schematic views of mouse skull with landmarks used for measuring linear distances.Anatomical description for each landmark is found in Table I (with permission from J. Richtsmeier: http://getahead.psu.edu/landmarks_new.html).

Table I Anatomical description of landmarks used for morphometric analysis

\section{Point Anatomical description}

A Nasale: Intersection of nasal bones, rostral point

B Nasion: Intersection of nasal bones, coudal point

C Bregma: intersection of frontal bones and parietal bones at midline

Intersection of parietal bones with anterior aspect of interparietal bone at midline

Intersection of interparietal bones with squamous portion of occipital bone at midline

F

Anterior-most point at intersection of premaxillae and nasal bones, left side

G

Anterior-most point at intersection of premaxillae and nasal bones, right side

$H \quad$ Anterior notch on frontal process lateral to infraorbital fissure, left side

Anterior notch on frontal process lateral to infraorbital fissure, right side

Intersection of zygoma (jugal) with zygomatic process of temporal, superior aspect, left side

K

Intersection of zygoma (jugal) with zygomatic process of temporal, superior aspect, right side

Intersection of parietal, temporal and interparietal bones, left side

M
Intersection of parietal, temporal and interparietal bones, right side 


\section{Geometric morphometric methods}

Differences in skull shape were analyzed using the 3D Landmark Coordinate Data and Euclidean Distance Matrix Analysis or EDMA. EDMA is a coordinate system-free method for statistically evaluating differences in size and shape of biological objects. EDMA converts 3D landmark data into linear distances, compiling a matrix of all linear distances between unique landmark pairs. In our samples, we used a customized EDMA version to calculate the distance in millimeters converted from the pixel size in the mCT scans. The distances between the landmarks were calculated by using the $\mathrm{X}, \mathrm{Y}$ and $\mathrm{Z}$ coordinates of each landmark in the equation $\mathrm{d}=(\sqrt{ } \cdot(\mathrm{x} 2-\mathrm{x} 1) 2+(\mathrm{y} 2-$ $\left.\mathrm{y}^{1) 2+(z 2-\mathrm{z} 1) 2}\right)$. Angles between landmarks were determined using the "measure angle" function of Analyze software.

\section{Histological sections}

Tissues were harvested after euthanasia and fixed with 4\% paraformaldehyde, followed by demineralization with 0.5 M EDTA. Samples were embedded in paraffin and $5 \mu \mathrm{m}$ sections prepared for histologic analysis. Sections were placed on poly L-lysine coated slides, dried overnight and stained with H\&E.

\section{Statistical analysis}

Statistical significance was assessed by Student's t-test. Values were considered statistically significant when $p \leq 0.05$. To assess the accuracy of the landmarking process, we placed 7 landmarks (A-E, $\mathrm{L}$ and $\mathrm{M}$ ) on one skull and repeated the process 10 times. The overall median variability was $0.128 \mathrm{~mm}$ with a maximum of $0.316 \mathrm{~mm}$ $(\mathrm{Q} 1=0.092 \mathrm{~mm}, \mathrm{Q} 3=0.163 \mathrm{~mm})$. This precision is similar to those reported for manual placement of landmarks mouse skulls. ${ }^{22,23}$

\section{Results and discussion}

Direct comparison of Nflob-/- and control using the complete set of landmarks, showed several statistically significant differences in the linear distance between Nflob-/- sample and controls. Analysis of occlusion revealed that, Nf1ob-/- mice have a higher tendency to develop malocclusion of incisor teeth (Figure 2) as by 12 weeks of age $26 \%$ in Nflob-/- mice exhibit frank malocclusion of incisors. One hundred percent of animals were found to exhibit malocclusion by 48 weeks of age. The incidence of malocclusion in the C57BL/6 mouse genetic background is reported to be $0.046 \%{ }^{24,25}$ The increased frequency of malocclusion in Nflob-/- mice relative to control mice parallels dental abnormalities among NF1 patients. ${ }^{26}$ In many cases of craniofacial dysplasia in NF1 a plexiform neurofibroma or optic nerve glioma is found adjacent to the bone, which may drive dysplastic changes in bone. To rule out tumor involvement in the Nflob-/- mouse craniofacial defect we histologically examined, severe examples of malocclusion, facial asymmetry and proptosis of an eye. We found no associated tumors in these specimens (data not shown).

Analysis of the nasal region showed that the anteroposterior linear distance (between landmarks A and B) to be shorter in Nf1ob-/mice (compared control animals). However, there was no significant difference at the posterior region of the skull (i.e. the distance between landmarks $\mathrm{B}$ and E). There were no significant differences in the shape of the zygomatic bone region (landmarks H, I, J, and K) between Nf1ob-/- and control mice (Figure 3A \& 3B). We measured the angle of deviation from the midline as defined by a line drawn through landmarks B, C, D, and E to test for asymmetry. Deviation from the midline of the landmarks representing the nasal and premaxilla (landmarks A, F, and G), revealed this region in Nflob-/- mice to be significantly deviated $(3.30 \pm 1.47 \mathrm{o})$ from the midline compared to control animals $(0.204 \pm 0.150)(\mathrm{p}<0.0001)$. Nasal and premaxilla of 8 animals were deviated to the left and 2 animals deviated to the right, with greatest angle of $6.7 \mathrm{o}$ in Nf1ob-/- mice compared to the greatest angle of $0.4 \mathrm{o}$ in control mice (Figure 3D \& 3E). In the neurocranium region the linear distances oriented along the mediolateral axis (landmarks $\mathrm{L}$ and $\mathrm{M}$ ) and the distance along the rostro-caudal axis (landmarks $\mathrm{C}$ and E) showed no significant difference between Nflob-/- and control mice (Appendix Figure 1A). A trend toward macrocephaly is however present in Nflob-/- mice with an average distance between landmarks $\mathrm{L}$ and $\mathrm{M}$ of $9.28 \pm 1.7 \mathrm{~mm}$ compared to $8.06 \pm 0.57 \mathrm{~mm}$ in control (Appendix Figure 1). From the lateral aspect, the linear distance between landmarks $\mathrm{A}$ and $\mathrm{E}$ was reduced in Nflob-/- mice, as mentioned previously, however the distance between landmarks $\mathrm{B}$ and $\mathrm{E}$ as well as $\mathrm{C}$ and $\mathrm{E}$ were similar in Nflob-/- and control mice. This gives an indication that the disturbance in the anteroposterior dimension of the skull in Nflob-/-mice was confined to the frontal region of the skull. Moreover, measuring the angle between two lines (i.e. the first line passing through landmark A and B; and the second one between $\mathrm{C}$ and D), showed that the Nf1ob-/- mice display a more acute angle with an average of $138.2 \pm 3.17 \mathrm{o}$ compared to control mice at $146.8 \pm 2.17 \mathrm{o}$ (Figure 4 ). These measurements were significantly different between Nflob-/- mice and control $(\mathrm{p}<0.0001)$ (Figure $4 \mathrm{c}$ ). Thus, the nasal and premaxilla bones are tipped downward in Nflob-/- mice while control mice showed a more flattened, or straight arrangement antero-posteriorly.
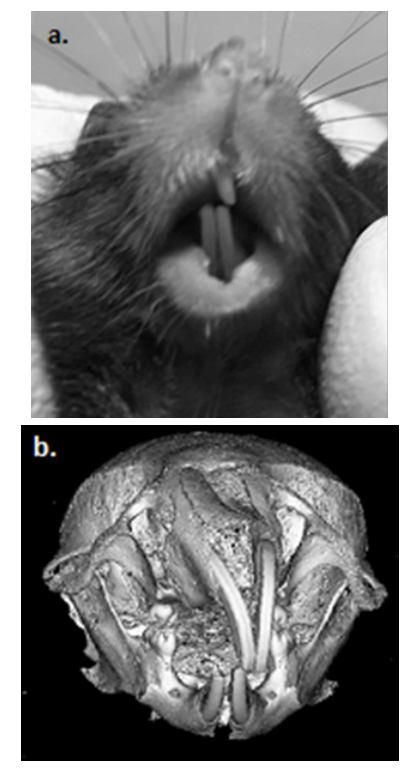

C

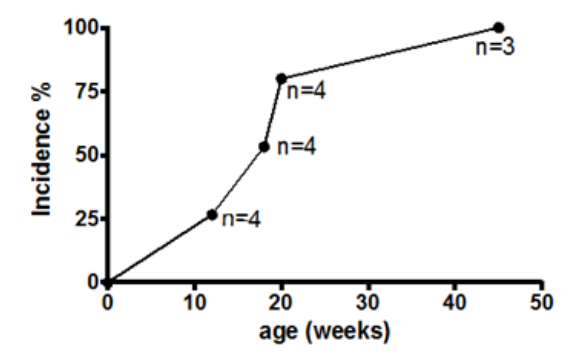

Figure 2 (A) Malocclusion of incisors in $\mathrm{NfI}_{\circ \mathrm{b}}{ }^{-/-}$mouse. a. Incisor malocclusion in an $\mathrm{NfI}_{\text {ob }}-/$ - mouse ( 16 weeks of age); (B) $\mu \mathrm{CT}$ frontal view of mouse skull confirms the deviation from the midline, resulting in incisor malocclusion, the occlusion of molars have not been evaluated; (C) The time-course of progressive overt phenotype of malocclusion in the $\mathrm{NfI}_{\text {ob }}-/-$ mice $(n=\mid 5)$. 

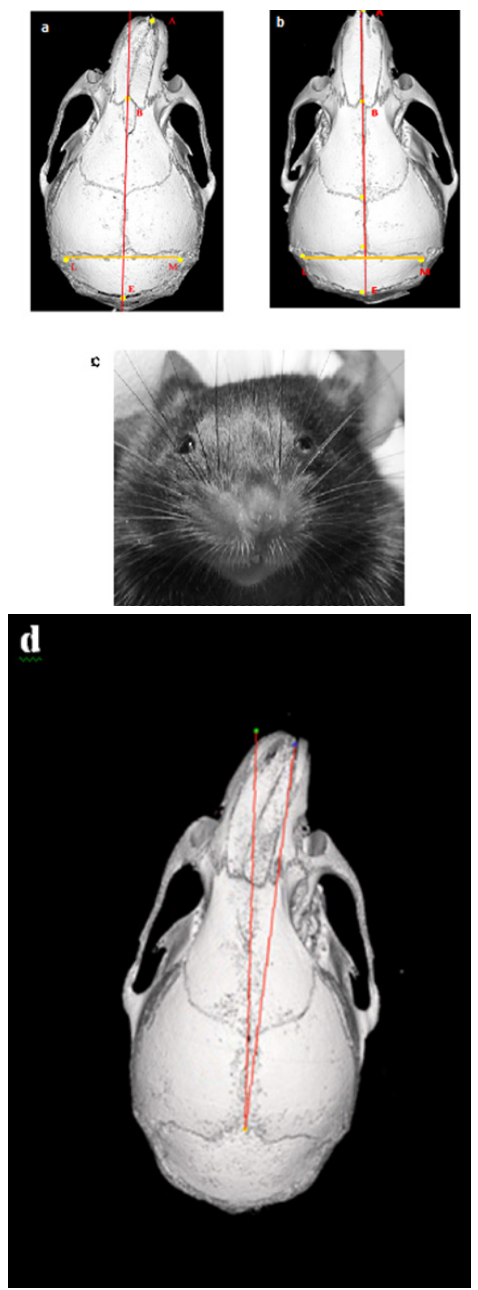

e

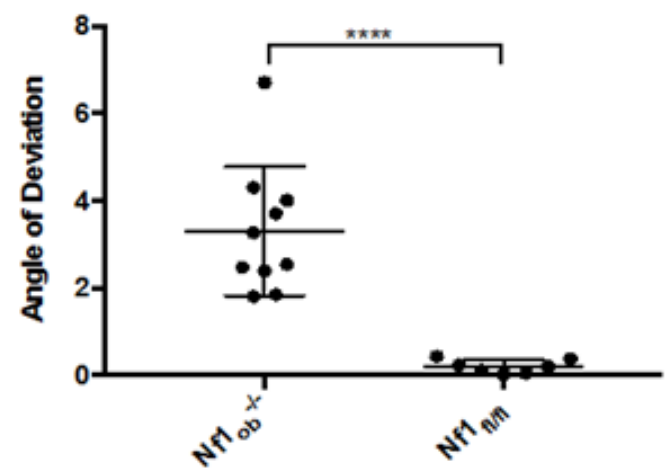

Figure 3 Facial deformity and midline deviation in $\mathrm{Nfl}_{{ }_{o b}} /$ - mice. Superior view of I4-week old mouse cranium (A) $\mathrm{NfI}_{0}^{-/}$- mouse, showing shorter linear distance between points $A$ and $B$, and nasal bones deviated from the midline (red line), when compared to control mouse; (B) $\mathrm{NfI}_{\text {ffft }}$ mouse. (C) Anterior view of $16 \mathrm{~W}$ old mouse face showing deviation from the midline and proptosis of an eye, which is a typical presentation of the craniofacial deformity in NFI. (D) Superior view of I4-week old $\mathrm{Nfl}_{\text {ob }}$-/- mouse cranium showing deviation of the anterior part of the skull from the midline. The red lines showing the angle between Nasale (landmark A) and the midline (i.e. straight line passing through points; $\mathrm{C}$ and D). (E) Variation in midline in $\mathrm{NfI}_{\mathrm{ob}} / /$ - and control $\mathrm{NfI}_{\mathrm{fl}}$ ff mice showed a significant difference with $p<0.0001$.
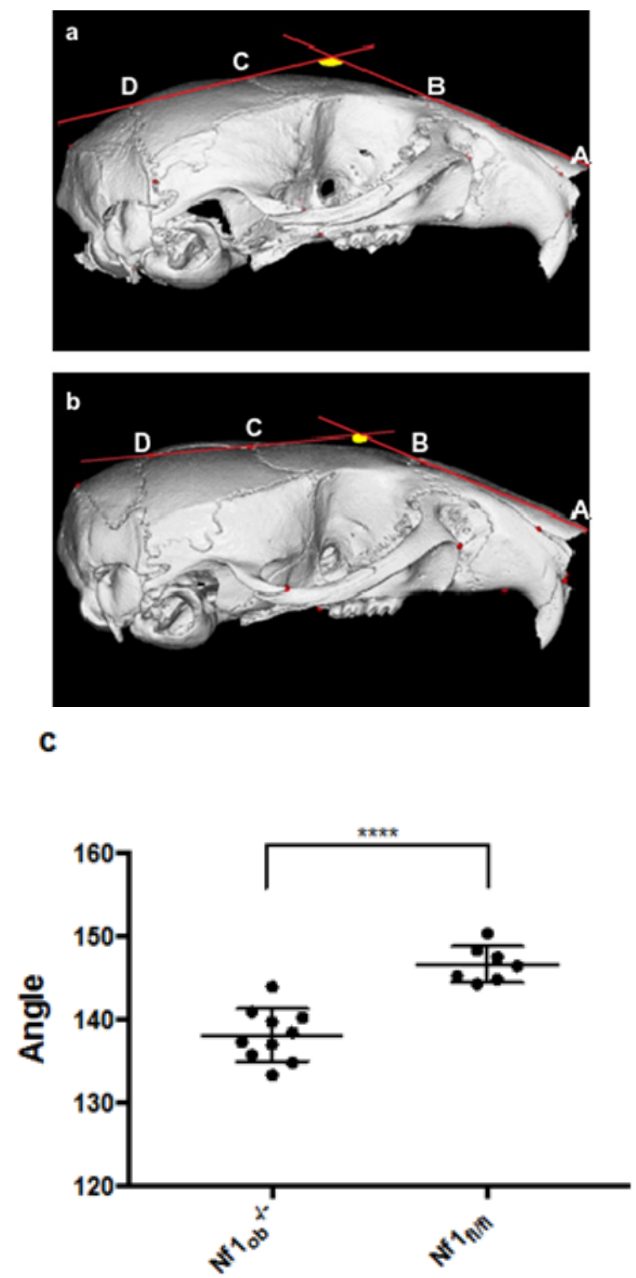

e

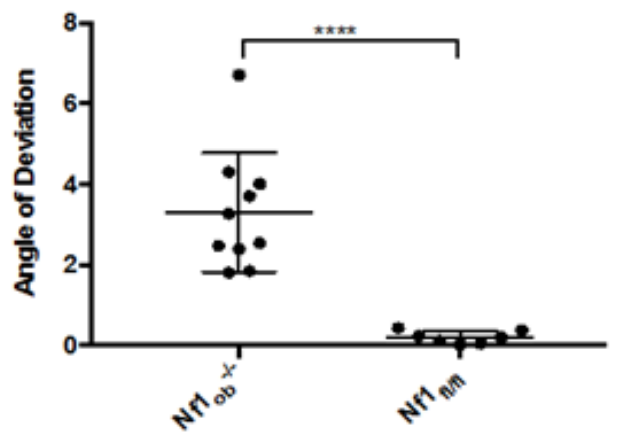

Figure 4 The craniofacial deformity in $\mathrm{NfI} \%$ - mice from a lateral view. (A) $\mathrm{NfI}_{\mathrm{ob}}-/$ - mouse cranium (I4 weeks of age), showing more acute angle between two lines (first, between $A$ and $B$; and second, between $C$ and $D$ ), when compared to control $\mathrm{NfI}_{\mathrm{f} / \mathrm{f}}$ mouse. (B) This indicates that the rostrum, or face, demonstrates klinorhynchy or a downward tipping face in $\mathrm{Nfl}^{-}-$- mice which contributes to the resultant facial deformity. (C) Nasal bones are tipped downward in $\mathrm{NfI}_{\mathrm{ob}} /$ - mice. Graph showing that $\mathrm{NfI}_{\mathrm{ob}} /$ - mice have a more acute angles of anterior part of the skull relevant to the neurocranium with an average of $138.2^{\circ}$ compared to control $\mathrm{NfI}_{\mathrm{ffff}}$ mice with an average of $146.8^{\circ}$. The difference in angle between $\mathrm{NfI}_{o b}{ }^{-/}$and control mice was statistically significant with $\mathrm{p}<0.0001$. 

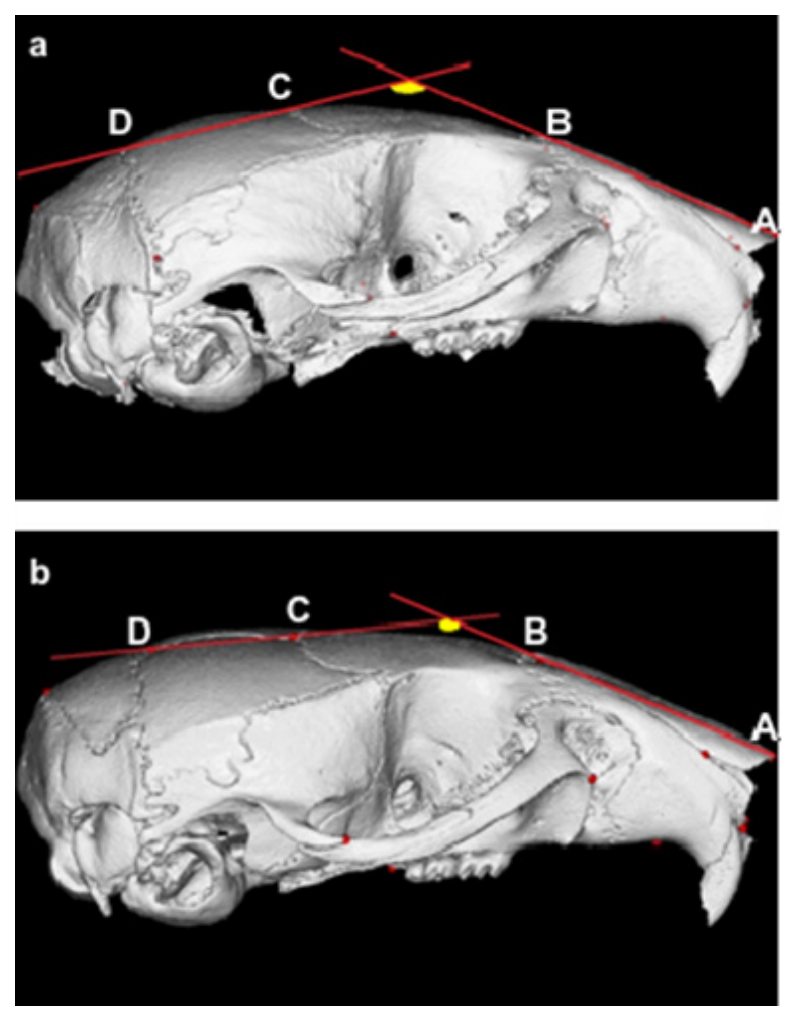

C

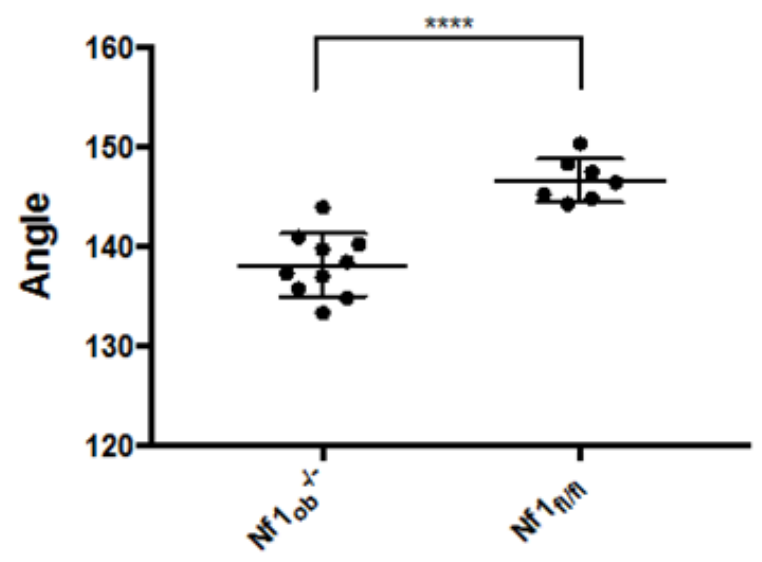

Appendix Figure I A trend toward macrocephaly in $\mathrm{Nfl}_{\mathrm{ob}} /-$ mice. (A) Superior view of mouse skull showing the distance measured to analyze the neurocranium. (B) Average neurocranium in $\mathrm{Nfl}_{\mathrm{ob}}{ }^{-/}$mice is slightly larger. The difference is not statistically significant compared to control however, it indicates a trend toward macrocephaly.

In this study we showed that Nf1ob-/- mice develop facial deformity and malocclusion with osteoblast conditional deletion of the Nfl gene. The phenotype is progressive, is unilateral, and often results in proptosis of an eye. This phenotype is strikingly similar to sphenoid wing dysplasia in NF1, which positions these animals as models with which to test interventions and mechanisms. The skeletal abnormalities in NF1 and their molecular basis has been difficult to study, in part because $\mathrm{Nf1}(+/-)$ mice do not display a skeletal phenotype and $\mathrm{Nf1(-/-)}$ mice die in utero before bone formation occurs. ${ }^{27-29}$ Elefteriou and colleagues in 2006 generated mice lacking Nf1 gene in osteoblast cells; the bone cells in which neurofibromin expression is the highest. ${ }^{19}$ This conditional deletion bypasses the embryonic lethality of a somatic deletion of the gene, generating a mouse model allowing for the analysis of the function of NF1 in bone and further study of the resulting skeletal abnormalities. No axial or appendicular skeletal abnormalities are seen in $\mathrm{Nfl}_{\mathrm{ob}}{ }^{-/-}$ mice. They do however present with a high bone mass phenotype with increased osteoblast numbers, increased bone formation and increased osteoclast numbers. We observed that $\mathrm{Nfl}_{\mathrm{ob}}-/$ - mice have a progressive facial asymmetry. $\mathrm{Nfl}_{\mathrm{ob}}-/$ - mice develop malocclusion and proptosis of an eye. In mice, malocclusion occurs when the incisors overgrow due to misalignment of the jaw. Therefore, the mandibular and maxillary teeth do not properly occlude. ${ }^{24,30}$ The incidence of malocclusion in the C57BL/6 mouse genetic background has been shown to be $0.046 \% .{ }^{24,25} \mathrm{We}$ found that 26 percent of $\mathrm{Nf}_{\mathrm{ob}}-/$ - mice on the C57BL/6 background, by 12 weeks of age animals displayed frank $\mathrm{Nfl}_{\mathrm{ob}} /-$ - malocclusion of the incisors. One hundred percent these mice displayed malocclusion by 48 weeks of age (Figure 2). Malocclusion frequently accompanies sphenoid wing dysplasia in NF1. ${ }^{31}$ and it has been proposed that the incidence of asymmetry in the midface region and sphenoid wing dysplasia may be much higher if detailed radiographic studies of asymptomatic NF1 patients are performed..$^{32}$ Using geometric morphometric analysis, we show that $\mathrm{Nfl}_{\mathrm{ob}^{-}} /-$mice develop asymmetry of the nasal region. As shown in Figure 3A \& $3 \mathrm{C}$, the nasal bones were found to diverge significantly from the long axis of the cranium. This dysplasia was found to be either to the left or right and was independent of sex. Additionally, the nasal region in $\mathrm{Nfl}_{\mathrm{ob}}-/-$ mice was found to demonstrate klinorhynchy or be tipped downward to a significant extent (Figure 4).

In NF1, the most clinically striking craniofacial change is facial asymmetry and dysplasia of the sphenoid bone resulting in unilateral eye proptosis. ${ }^{33}$ Computed tomography (CT) imaging reveals enlargement of middle cranial fossa with sphenoid bone changes. While cranial bones develop from analogous structures and by similar mechanisms between mouse and human, mouse cranial bone morphology is different from human. ${ }^{34}$ In mice, the eyes are not in orbits as such but in more of a channel formed by the zygomatic arch. The sphenoid bone does not form a large portion of the orbits as in humans. The eyes also are on the side of the head and point more up and out rather than forward as in the human. We find significant and robust changes in the nasal region of Nflob-/- mice. This pattern of deformed frontal midface region of the skull in Nflob-/- mice parallels the quantitative changes in the analogous structures in human NF1 craniofacial skeleton resulting in unilateral proptosis of an eye and facial asymmetry. ${ }^{12-26}$ NF1 patients often exhibit macrocephaly. We find a weak trend toward macrocephaly that does not rise to statistical significance (Supplementary Figure 1). It is possible that the pathways and signaling in of neurofibromin the neurocranium is different from that of the viscerocranium or if the mechanism of macrocephaly in NF1 is not osteoblast dependent.

It has been hypothesized that the dysplasia of sphenoid bone is unique and not like other bony abnormalities in NF1 and has been proposed to originate secondary to plexiform neurofibromas (PNF) which affect the nearby bone structure. ${ }^{33}$ We find no tumoral involvement in the Nflob-/- mice with malocclusion and proptosis of an eye. In addition, expression of the of $2.3 \mathrm{~kb}$ coll $\alpha 1$ promotor-driven Cre recombinase has not been reported in neural tissues. Therefore, 
due to the primarily osteoblast expression of the of $2.3 \mathrm{~kb}$ coll $\alpha 1$-Cre, our data support an osteoblast origin for sphenoid wing dysplasia and craniofacial asymmetry in the absence of tumor in NF1. It has been proposed that the skeletal dysplasia in NF1 is dependent upon a defect in mechanical signaling in bone cells. ${ }^{35}$ The differentiation and function of osteoblasts and osteoblast-lineage cells is well known to be modulated by mechanical signaling. Mechanical signaling in osteoblast cells has in turn been shown to occur via signaling through the RAS pathway, which is negatively regulated by the neurofibromin protein. ${ }^{36-38}$ In mice the largest mechanical forces encountered are likely masticatory forces present while chewing. Interestingly, bones and muscles involved in mastication are those primarily affected in $\mathrm{Nfl}_{\mathrm{ob}}{ }^{-/-}$animals. Indeed, sphenoid wing bones anatomically articulate with maxillary and zygomatic bones and the neurocranium, ${ }^{39,40}$ which is less affected in the $\mathrm{Nf}_{\mathrm{ob}^{-}}-/$- model.

\section{Conclusion}

In conclusion, we have identified a mouse model of craniofacial dysplasia in NF1, which will help identify the molecular mechanism and pathways responsible for the craniofacial deformity in NF1 syndrome. There are currently no treatments known that block or slow the progression of sphenoid wing dysplasia in NF1. The Nf1ob-/mouse represents a novel and relevant mouse model that recapitulates the phenotype of sphenoid wing dysplasia in NF1. Nflob-/- mice provide an important tool with which to test treatments to prevent facial deformities in pediatric NF1 patients.

\section{Acknowledgments}

None.

\section{Conflicts of interest}

The authors declare there is no conflict of interest.

\section{References}

1. Tonsgard JH. Clinical manifestations and management of neurofibromatosis type 1. Semin Pediatr Neurol. 2006;13(1):2-7.

2. Chrcanovic BR, Gomez RS, Freire-Maia B. Neurofibromatosis type 1 associated with bilateral central giant cell granuloma of the mandible. $J$ Craniomaxillofac Surg. 2011;39(7):538-543.

3. Heerva E, Peltonen S, Pirttiniemi P, et al. Short mandible, maxilla and cranial base are common in patients with neurofibromatosis 1. Eur J Oral Sci. 2011;119(2):121-127.

4. Vitale MG, Guha A, Skaggs DL. Orthopaedic manifestations of neurofibromatosis in children: an update. Clin Orthop Relat Res. 2002;401:107-118.

5. Stevenson DA, Zhou H, Ashrafi S, et al. Double inactivation of NF1 in tibial pseudarthrosis. Am J Hum Genet. 2006;79(1):143-148.

6. Ruggieri M, Pavone V, De Luca D, et al. Congenital bone malformations in patients with neurofibromatosis type 1 (Nf1). J Pediatr Orthop. 1999;19(3):301-315.

7. Sabbagh A, Pasmant E, Laurendeau I, et al. Unravelling the genetic basis of variable clinical expression in neurofibromatosis 1. Hum Mol Genet. 2009;18(15):2768-2778.

8. Sabbagh A, Pasmant E, Imbard A, et al. NF1 molecular characterization and neurofibromatosis type I genotype-phenotype correlation: the French experience. Hum Mutat. 2013;34(11):1510-1518.
9. Rhodes SD, Zhang W, Yang D, et al. Dystrophic spinal deformities in a neurofibromatosis type 1 murine model. PLoS One. 2015;10(3):e0119093.

10. Virdis R, Street ME, Bandello MA, et al. Growth and pubertal disorders in neurofibromatosis type 1. J Pediatr Endocrinol Metab. 2003;16 Suppl 2:289-292.

11. Clementi M, Milani S, Mammi I, et al. Neurofibromatosis type 1 growth charts. Am J Med Genet. 1999;87(4):317-323.

12. Akadiri IJ. Craniofacial Neurofibromatosis Type 1: clinical features, challenges of management, and a report of 2 Nigerian patients. The Internet Journal of Head and Neck Surgery. 2008;3(1):1-7.

13. Bardellini E, Amadori F, Flocchini P, et al. Oral findings in 50 children with neurofibromatosis type 1. A case control study. Eur J Paediatr Dent. 2011;12(4):256-260.

14. Visnapuu V, Peltonen S, Tammisalo T, et al. Radiographic findings in the jaws of patients with neurofibromatosis 1. J Oral Maxillofac Surg. 2012;70(6):1351-1357.

15. Friedrich RE, Giese M, Schmelzle R, et al. Jaw malformations plus displacement and numerical aberrations of teeth in neurofibromatosis type 1: a descriptive analysis of 48 patients based on panoramic radiographs and oral findings. Journal of Cranio-Maxillofacial Surgery. 2003;31(1):1-

16. Kuorilehto T, Pöyhönen M, Bloigu R, et al. Decreased bone mineral density and content in neurofibromatosis type 1 : lowest local values are located in the load-carrying parts of the body. Osteoporos Int. 2005;16(8):928-936.

17. Lammert M, Kappler M, Mautner VF, et al. Decreased bone mineral density in patients with neurofibromatosis 1. Osteoporos Int. 2005;16(9):11611166.

18. Dacquin R, Starbuck M, Schinke T, et al. Mouse alpha1 (I)-collagen promoter is the best known promoter to drive efficient Cre recombinase expression in osteoblast. Dev Dyn. 2002;224(2):245-251.

19. Elefteriou F, Benson MD, Sowa H, et al. ATF4 mediation of NF1 functions in osteoblast reveals a nutritional basis for congenital skeletal dysplasiae. Cell Metab. 2006;4(6):441-451.

20. Wang W, Nyman JS, Ono K, et al. Mice lacking Nf1 in osteochondroprogenitor cells display skeletal dysplasia similar to patients with neurofibromatosis type I. Hum Mol Genet. 2011;20(20):3910-3924.

21. (2016) Getahead.psu.edu. Landmarks.

22. Paul AB. Semi-automatic landmark point annotation for geometric morphometrics. Frontiers in Zoology. 2014;11(1):61.

23. Aneja D, Vora SR, Camci ED, et al. Automated Detection of 3D Landmarks for the Elimination of Non-Biological Variation in Geometric Morphometric Analyses. Proc IEEE Int Symp Comput Based Med Syst. $2015 ; 78-83$.

24. Petznek H, Kappler R, Scherthan H, et al. Reduced body growth and excessive incisor length in insertional mutants mapping to mouse Chromosome 13. Mamm Genome. 2002;13(9):504-509.

25. Saito F, Kajii TS, Sugawara Kato Y, et al. Three-dimensional craniomaxillary characteristics of the mouse with spontaneous malocclusion using microcomputed tomography. Eur J Orthod. 2011;33(1):43-49.

26. Beck M, Peterson JF, McConnell J, et al. Craniofacial abnormalities and developmental delay in two families with overlapping 22q12.1 microdeletions involving the MN1 gene. Am $J$ Med Genet $A$. 2015;167A(5):1047-1053.

27. Jacks T, Shih TS, Schmitt EM, et al. Tumour predisposition in mice heterozygous for a targeted mutation in Nf1. Nat Genet. 1994;7(3):353361 . 
28. McClatchey AI, Cichowski K. Mouse models of neurofibromatosis Biochim Biophys Acta. 2001;1471(2):M73-M80.

29. Brannan CI, Perkins AS, Vogel KS, et al. Targeted disruption of the neurofibromatosis type-1 gene leads to developmental abnormalities in heart and various neural crest-derived tissues. Genes Dev. 1977;8(9):10191029.

30. Miller WA. Genetic traumatic occlusion in the mouse. J Periodontal Res. 1977;12(1):64-72.

31. Stevenson DA, Viskochil DH, Carey JC. Neurofibromatosis type 1 is a genetic skeletal disorder. Am J Med Genet A. 2007;143A(17):2082-2083.

32. Sigillo R, Rivera H, Nikitakis NG, et al. Neurofibromatosis type 1: a clinicopathological study of the orofacial manifestations in 6 pediatric patients. Pediatr Dent. 2002;24(6):575-580.

33. Claude Jacquemin T, Helena Svedberg. Orbit Deformities in Craniofacial Neurofibromatosis Type 1. American Journal of Neuroradiology. 2003;24(8):1678-1682.

34. Verdyck P, Wuyts W, Van Hul W. Genetic defects in the development of the skull vault in humans and mice. Crit Rev Eukaryot Gene Expr. 2006;16(2):119-142.
35. Alwan S, Tredwell SJ, Friedman JM. Is osseous dysplasia a primary feature of neurofibromatosis 1 (NF1)? Clin Genet. 2005;67(5):378-390.

36. Rubin J, Murphy TC, Rahnert J, et al. Mechanical inhibition of RANKL expression is regulated by H-Ras-GTPase. $J$ Biol Chem. 2006;281(3):1412-1418.

37. Rubin J, Murphy TC, Fan X, et al. Activation of extracellular signalregulated kinase is involved in mechanical strain inhibition of RANKL expression in bone stromal cells. J Bone Miner Res. 2002;17(8):14521460 .

38. Yu X, Chen S, Potter OL, et al. Neurofibromin and its inactivation of Ras are prerequisites for osteoblast functioning. Bone. 2005;36(5):793-802.

39. Tortora GJ. Atlas of the human skeleton: updated to accompany Principles of anatomy and physiology 10/E. 3rd ed. New York, USA: Wiley \& Sons; 2003:1-26.

40. Claude Jacquemin B, Don Liu, Helena Svedberg, et al. Reassessment of Sphenoid Dysplasia Associated with Neurofibromatosis Type 1. American Journal of Neuroradiology. 2002;23(4):644-648. 